\title{
Bemerkungen zu der Abhandlung E. Krompechers: „Zur Kenntnis der Geschwülste und Hypertrophien der SchweiBdrüsen."
}

\author{
Von Prof. G. Ricker in Magdeburg.
}

Die im Titel genannte Abhandlung E. K rompechers, ${ }^{1}$ ) geeignet falsche Vorstellungen von den in meiner gemeinsam mit Johannes Schwalb verfaßten Monographie über die Geschwülste der Hautdrüsen ${ }^{2}$ ) vertretenen Anschauungen insbesondere über die Hidradenome, zu geben und bemüht dieselben $\mathrm{zu}$ widerlegen, zwingt mich zu einigen kurzen Bemerkungen der Richtigstellung und Abwehr.

$\mathrm{Kr}$ om pech er behauptet, wir hätten in unserer Monographie die Epitheliome und Karzinome der Haut im allgemeinen auf ihre Beziehungen zu den Hautdrüsen geprüft und als Resultat die These aufgestellt, "daß sozusagen sämtliche Basalzellentumoren der Haut Talgdrüsen- und Schweißdrüsentumoren entsprechen “. Diese Behauptung trifft nicht zu; wir haben die Epidermisgeschwülste ganz unerörtert gelassen und nur nachgewiesen, daB die unter der Epidermis, im Korium und Unterhautfett gelegenen und daselbst entstehenden epithelialen Geschwülste, von denen viele, namentlich auch von Krompecher, ohne Beweis als Karzinome aufgefaßt, von uns als Epitheliome bezeichnet worden sind, nicht - mit Krompecher von der Epidermis abzuleiten, sondern Geschwülste der

1) Dieses Archiv Bd. CXXVI. p. 765.

2) G. Ricker und Johannes Schwalb, die Geschwülste der Hautdrüsen, Berlin 1914, Verlag von S. Karger. 
Ricker. Zur Kenntnis der Geschwũlste und Hypertrophien etc. $\mathbf{3 0 3}$

Hautdrüsen sind. An die Stelle der demgemäß für diese Geschwülste unzutreffenden Bezeichnung Krompechers "Basalzellgeschwülste" - Geschwülste, ausgegangen von den basalen Zellen der Epidermis - haben somit die passend gewählten Namen der Talg- und Schweißdrüsengeschwïlste zu treten.

In seinem Bestreben diese Beweisführung zu widerlegen, geht Krompecher davon aus, daß die fertige Epidermis außer Leisten und Zapfen Talgdrüsen oder solchen älnnliche Gebilde bervorbringen könne; wir haben das (auf p. 40) anerkannt und verwertet. Wenn aber Krompecher angibt, dab die fertige Epidermis unter pathologischen Umständen auch schweißdrüsenähnliche Schläuche hervorzubringen vermag, so sehen wir diesen Nachweis nirgends, auch nicht bei Krompecher, erbracht. Seltsamerweise führt Krompecher zum Beweis in der Monographie auch von uns ausführlich und zustımmend erörterte Beispiele von Sprossenbildung der Schweißdrüsenausführungsgänge an; und nachdem er fortgefahren: „A uch ich selbst beobachtete öfters, wie die Basalzellenschicht des Oberflächenepithels in Form langer, dünner resp. enger Schläuche wucherte ......, welche demnach an Ausführungsgänge der Schweißdrüsen erinnern, " zieht er unmittelbar darauf den folgenden Schluß: "Alle diese Beobachtungen zeigen also, daß die Basalzellensehicht der Epidermis des Deckepithels schon an und für sich den Talg- und Schweißdrüsen ähnliche verschiedenartige Gebilde erzeugen kann." Wir entgegnen hierauf, daß was den Talgdrüsen recht ist, den Schweißdrüsen noch lange nicht billig zu sein braucht und ist, daß die Wucherung der Schweißdrüsenausführungsgänge nichts für das gleiche Wucherungsvermögen der Epidermis beweist, und daß die "ofters" gemachte Beobachtung Krompechers bisher von ihm nicht in einer die Prüfung ermöglichenden Form mitgeteilt ist; die bloße Angabe, zumal in der Weise, wie sie gegeben wird (Beschreibung eines der Beobachtang entzogenen Vorganges !), ist nicht im Stande, zu beweisen, daß die fertige Epidermis unter pathologischen Bedingungen schweißdrüsenähnliche 
Gebilde, Schläuche hervorbringen kann. Wir bleiken also dabei, daß von einer solchen Fähigkeit der Epidermis bisher nichts bekannt ist, und dasselbe gilt von dem von K rompecher behaupteten Vermögen der - hypothetischen - sogenannten „versprengten Deckenepithelkeime", solche Schläuche zu bilden.

Auf die Geschwülste aus typisch und annähernd typisch hyperplastischen Schweißdrüsen, auf die einfachen und die komplizierten (papillären) zystischen Geschwülste der Schweißdrüsen brauchen wir nicht einzugehen, da Krompecher hierzu nichts Neues bringt; es sei nur bemerkt, daß die papilläre Form nicht, wie Krompecher angibt, von ihm aufgestellt worden ist, und daß wir es nicht für angängig halten, zystische und papillärzystische Geschwülste, wie $\mathrm{Kr}$ om pecher, zu den Adenomen zu rechnen. Dagegen haben wir uns mit den Adenomen der Schweißdrüsen zu beschäftigen; wir verstehen darunter diejenigen Geschwülste, deren Parenchym aus Schläuchen besteht, nicht in typischem Aufbau, wie bei der typischen Hyperplasie, auch nicht in atypischer Anordnung und Verbreitung wie beim Adenokarzinom, sondern in, wie wir es genannt haben, paratypischer Form, die in den einzelnen in der Literatur behandelten Geschwülsten sehr verschieden gewesen ist.

Krompecher spricht -.- nach dem Vorgange von Ludwig $\mathrm{Pick} \mathrm{k}^{1}$ - von Hidradenom und Adenoma hidradenoides; unter Hidradenomen versteht er Adenome, „welche nachweislich von fertigen, wohlausgebildeten Schweißdrüsen ausgehen, unter hidradenoiden Adenomen solche, welche wohl die charakteristischen Eigenschaften von Schweißdrüsenadenomen aufweisen, aber nicht von fertigen Schweißdrüsen, sondern von rudimentären Schweißdrüsen oder vom Oberepithel der Epidermis ausgehen".

Als Hidradenome in diesem Sime behandelnd erkennt K rompecher vier Mitteilungen in der Literatur an, die von Thierfelder, W. Petersen, Brauns und Thimm. Iñdem wir auf die ausführliche Besprechung dieser

1) Ludwig Pick, Über Hidradenoma und Adenoma hidradenoides, Virchows Archiv, CLXXV. Bd., 1904. 
Abhandlungen in unserer Monographie hinweisen, beschränken wir uns hier darauf, hervorzuheben, daß die von Thierfeld er beschriebene Geschwulst kein Adenom, sondern ein Adenokarzinom gewesen, und daß die Geschwulst nicht mit Knäueldrüsen zusammengehangen hat (ein solcher Zusammenhang mit unanfechtbaren Schweißdrüsen muß nach Krompecher beim Hidradenom vorhanden sein); daß Brauns und Thimm im wesentlichen zystisch, daneben typisch hyperplastische Schweißdrüsen beschrieben haben, Neubildungen, die unserer Auffassung nach nicht als Adenome bezeichnet werden können; schließlich, daß von den multiplen Geschwülsten des 20jährigen Mädchens, die W. Petersen behandelt, nur ein Teil den K rompecherschen Anforderungen an ein "Hidradenom" gerecht wird (während Krompecher die übrigen Geschwülste als hidradenoide Adenome bezeichnen müBte).

Es ergibt sich somit, daB bei einer sorgfältigeren Prüfung der von den Autoren gegebenen Beschreibungen und einer strengeren Fassung des Adenombegriffes $\mathrm{K}$ r o mpecher auch noch jene vier, seiner Meinung nach einzigen von fertigen Schweißdrüsen ausgegangene Adenome als solche aufgeben und zu dem Satze hätte gelangen müssen, daß a 11 e Schweißdrüsenadenome hidradenoide Geschwülste, d. h. - nach Krompecher - von der Epidermis oder von "rudimentären“ (in der fetalen Entwicklung gestörten) Schweißdrüsen abzuleiten seien.

Vergeblich sucht man in K rompecher s Abhandlung nach einem Beweis für seine Auffassung. Es bleibt uns daher nur übrig, uns an Ludwig Pick zu halten, auf den sich Krompecher beruft, und nachzusehen, wie er sich zu der Frage nach dem Ursprunge der Schweißdrüsen. adenome stellt. P i ck zieht aus seinen ausführlichen Auseinandersetzungen den Schluß, daß man zu der Annahme "der kongenitalen Anlage sämtlicher bisher bekannter Fälle von Hidradenom und Adenoma hidradenoides in Form von Entwicklungsstörungen an den Schweißdrüsenanlagen gelangen müsse" ; Pick gibt also das aus fertigen Schweißdrüsen hervorgehende Adenom -- mit kleiner Einschrản- 
kung - völlig preis. Den Ausgang von Schweißdrüsenadenomen von der fertigen Epidermis zieht $\mathrm{Pick}$ überhaupt nicht in Betracht.

Krompecher beruft sich somit mit Unrecht auf $\mathrm{Pick}$, und unzutreffenderweise erweckt or in dem Leser seiner Abhandlung den Eindruck, daß Pick Schweißdrüsenadenome von der "Epidermis" schlechthin abgeleitet habe. Für die Ableitung von der fertigen Epidermis, für die sich Krompecher erklärt, fehlt vielmehr in der Literatur jeder Anhalt. Schweißdrüsenadenome gehen somit nicht von der fertigen Epidermis aus.

Bleibt noch die Ableitung von in ihrer (fötalen) Entwicklung gestörten Schweißdrüsenanlagen. Hier müßten wir uns mit Pick auseinandersetzen, nicht mit Krompecher, der keinerlei Gründe für diese Auffassung herbeibringt. Wir beschränken uns darauf anzuführen, daß wir in unserer Monographie den Zusammenhang von Schweißdrüsenadenomen mit fötalen Entwicklungsstörungen, wo es uns nötig schien, anerkannt, wo er ohne zwingenden Grund behauptet worden war, mit Einwänden bedacht haben; eine Verallgemeinerung, wie sie $\mathrm{Pick}$ vorgenommen, hielten und halten wir nicht für angebracht. Das Nähere ist in der Monographie zu finden; hier wollen wir an ihrer Hand nur darauf aufmerksam machen, daß wie vor, so auch nach Picks Abhandlung mehrere Mitteilungen über Schweißdrüsenadenome erschienen sind, die nicht zu der Annahme ihrer fötalen Anlage zwingen und die Autoren zur Ableitung von fertigen Schweißdrüsen bewogen haben, und ferner darauf, daß die hier in Betracht kommenden und von Pick besonders ergiebig verwerteten Geschwülste an den äuBeren Geschlechtsorganen des Weibes nicht ohne Bedenken von den Schweißdrüsen abgeleitet werden können. Wir können daher der Einteilung Picks nicht zustimmen, um so weniger, als er sie selbst untergräbt, indem er zwar Hidradenome aus fertigen Schweißdrüsen nicht als sicher nachgewiesen ansieht, aber selbst in Betracht zieht, daß ein "hidradenoides Adenom" unter Umständen auch von fertigen Schweißdrüsen ausgehen kann. Überdies sagt der Zusatz 
hidradenoid nichts über den Ausgangspunkt der Neubildung aus, sondern bedeutet lediglich schweißdrüsenähnlich, was, mehr oder minder, das Parenchym aller Schweißdrüsenadenome ist. Man spreche also weiterhin lediglich von Hidradenomen und erörtere, wenn eine Veranlassung dazu vorliegt, den Zusammenhang einer Neubildung mit den fötalen Wachtumsvorgängen, so wie andere Hautgeschwülste uns dazu bewogen haben, Wachstumsvorgänge im Kindesạlter, zur Zeit der Pubertät, im Greisenalter zur Anbahnung eines Verständnisses heranzuziehen; einen solchen Zusammenhang im Geschwulstnamen auszudrücken ist weder nötig noch sprachlich möglich.

Eine Einteilung der Hidradenome kann nach verschiedenen Gesichtspunkten erfolgen, so nach dem vorhandenen und fehlenden Zusammenhange mit der Epidermis, wie wir in unserer Monographie getan haben, oder nach dem verschiedenen, jedoch schon jetzt Typen verratenden Bau, eine Einteilung, die sich aber gut erst dann wird durchführen lassen, wenn die Zahl der sorgfältig beobachteten Hidradenome größer geworden und die Herkunft der an den Labien des Weibes zu findenden hidradenomoiden Geschwülste geklärt sein wird.

„Die Geschwülste werden (von Ricker und Schwalb) einfach ihrer Drüsenähnlichkeit wegen als Geschwülste der Hautdrüsen hingestellt." Wie unzutreffend diese Behanptung $\mathrm{K}$ r o m pech e r s ist, kann das Studium unserer Abhandlung lehren, bei dem der Leser auch prüfen mag, ob, wie dies $\mathrm{Kr}$ o m p e ch e r behauptet, unsere Einteilung die „sichere Grundlage" und unsere Bearbeitung der Hautdrüsengeschwülste „die verdiente Gründlichkeit" vermissen läßt. Wir haben uns, auch in bezug auf nnser eigenes umfangreiches Material, eifrig bemüht, dem rein Morphologischen sein volles kecht zu geben, darüber hinaus aber an der Hand der Physiologie und Pathologie die Geschwülste der Hautdrüsen zu beleuchten; wir glauben auf diese Weise, unter Heranziehung physiologisch-chemischer Kenntnisse, z. B. den von Krompecher in seiner uns hier beschäftigenden Abhandlnng unerwähnt gelassenen; von uns 
308 Ricker. Zur Kenntnis der Geschwülste u. Hypertrophien etc.

benannten muzinösen und chondrinösen Epitheliomen der Schweißdrüsen, Geschwülsten, denen gegenüber das im übrigen nicht zu verachtende Merkmal der Drüsenähnlichkeit völlig versagt, zu ihrer richtigen Auffassung verholfen zu haben, die allerdings von der von Krompecher (an anderer Stelle) vertretenen erheblich abweicht. Wollten wir uns in bezug auf diese und andere Punkte - wir erwähnen nur die sekundäre Vereinigung von epithelialen Formationen im Korium mit der Epidermis, die Umwandlnng ganzer Drüsen in Geschwülste und Geschwulstteile - in Erörterungen einlassen, zu denen die Krompechersche Abhandlung herausfordert, so müßten wir das Gebiet der allgemeinen Geschwulstlehre betreten, was in der hier gebotenen Kürze nicht möglich ist. Indem wir daher auf unsere Monographie, ferner auch auf $\mathrm{Ribberts}$ und Borrmanns zutreffende Kritik der Krompecherschen Ableitung der Koriumgeschwülste von der Epidermis verweisen, glauben wir aussprechen zu dürfen, daß unsere vorwiegend an fremden, verstreuten, und in weiten Kreisen, auch von $\mathrm{Krompecher}$, ungenügend berücksichtigten Beobachtungen, daneben durch eigene Untersuchungen gewonnene Hautdrüsengeschwülsten durch $\mathrm{Krompechers}$ Angriffe keine Widerlegung oder Anderung erfahren hat, - und daß somit für Krompechers "Basalzellentheorie“ auf dem Gebiete der Hautdrüsengeschwülste ebensowenig Platz ist, wie unserer Überzeugung nach an irgend einer anderen Stelle der Geschwulstlehre.

\section{Erwiderung auf die obigen Bemerkungen G. Rickers.}

\section{Von Prof. E. Krompecher in Budapest.}

Gegenüber der Behauptung Rickers, daß für meine "Basalzellentheorie" auf dem Gebiete der Hautdrüsengeschwälste ebensowenig Platz sei, wie an irgend einer anderen Stelle der Geschwulstlehre, brauche ich mich wohl 